\title{
A review on herbal antiasthmatics
}

\author{
Ravindra G. Mali • Avinash S. Dhake
}

Received: 27 January 2011 / Accepted: 1 April 2011 / Published online: 5 July 2011

(C) Institute of Oriental Medicine, Kyung Hee University 2011

\begin{abstract}
In traditional systems of medicine, many plants have been documented to be useful for the treatment of various respiratory disorders including asthma. In the last two decades the use of medicinal plants and natural products has been increased dramatically all over the world. Current synthetic drugs used in pharmacotherapy of asthma are unable to act at all the stages and targets of asthma. However some herbal alternatives employed in asthma are proven to provide symptomatic relief and assist in the inhibition of disease progression also. The herbs have shown interesting results in various target specific biological activities such as bronchodilation, mast cell stabilization, anti-anaphylactic, anti-inflammatory, anti-spasmodic, anti-allergic, immunomodulatory and inhibition of mediators such as leukotrienes, lipoxygenase, cyclooxygenase, platelet activating, phosphodiesterase and cytokine, in the treatment of asthma. This paper is an attempt to classify these pharmacological and clinical findings based on their possible mechanism of action reported. It also signifies the need for development of polyherbal formulations containing various herbs acting at particular sites of the patho-
\end{abstract}

R. G. Mali

L. B. Rao Institute of Pharmaceutical Education and Research, Shri B. D. Rao College Campus,

Khambhat 388 620, India

\section{A. S. Dhake}

S.M.B.T. College of Pharmacy, Dhamangaon,

Tal: Igatpuri,

Nashik 422 403, India

R. G. Mali $(\bowtie)$

Department of Pharmacognosy,

L. B. Rao Institute of Pharmaceutical Education and Research, Khambhat 388620 Gujarat, India

e-mail: ravigmali@yahoo.co.in physiological cascade of asthma for prophylaxis as well as for the treatment of asthma.

Keywords Asthma · Current therapy. Herbal therapy. Poly herbal formulations $\cdot$ Ayurvedic drugs $\cdot$ Medicinal plants

\section{Introduction}

According to the National Institute of Health (NIH), asthma is defined as a chronic inflammatory disorder of the airways in which many cells and cellular elements play a role, in particular, mast cells, eosinophils, T-lymphocytes, neutrophils and epithelial cells (NIH 1997). Asthma is caused by a very complex interaction between inflammatory cells and mediators. Herbal approaches have regained their popularity, for the treatment of asthma, with their efficacy and safety aspects being supported by controlled clinical studies (Huntley and Ernst 2000). Ongoing worldwide research has also provided valuable clues regarding the precise mechanism of action of these herbal alternatives (Goyal et al. 2007).

\section{Pharmacotherapy of bronchial asthma}

In the past most clinicians managed asthma mainly according to the patient's symptom. Asthma was regarded primarily as a problem of bronchospasm and measures to prevent or reverse bronchospasm comprised the mainstay of therapy. However, during early 1980s when asthma emerged as an inflammatory rather than primarily a bronchospastic disorder, the basic approach switched from control of symptoms to control of underlying airway inflammation (Barns 1989). According to guidelines of The National Asthma Education and Prevention Program's 
(NAEPP) guidelines for the diagnosis and management of asthma, the treatment should have following goals:

1. Maintain normal activity levels, including exercise.

2. Maintain normal or near normal pulmonary function.

3. Prevent chronic and troublesome symptoms.

4. Prevent recurrent exacerbations.

5. Avoid adverse effects from medications.

The pharmacological management of asthma depends upon frequency and severity of patient's symptoms. Infrequent attacks can be managed by treating each attack when it occurs, but with more frequent attacks preventive therapy needs to be used. The following categories of drugs are used in asthma:

\section{Bronchodilators}

$1.1 \beta$-adrenergic agonists: e.g. Metaproterenol, terbutaline, albuterol, formoterol, bitolterol, salmeterol, pirbuterol.

1.2 Anticholinergics: e.g. Ipratropium bromide, Tiotropium bromide.

1.3 Methylxanthines: e.g. Theophylline, aminophylline, acepiphylline, diprophylline, proxophylline.

2. Anti-inflammatory agents

2.1 Corticosteroids: e.g. Prednisolone, dexamethasone, beclomethasone dipropionate, dexamethasone, budesonide, fluticasone.

2.2 Anti-leukotrienes: e.g. Probilukast, Iralukast, Zieluton, Montelukast, zafirlukast, pranlukast.

2.3 Mast Cell Stabilizers: e.g. Cromolyn Sodium, Nedocromil sodium.

\section{Bronchodilators}

Bronchodilator drugs have an anti-bronchoconstrictor effect that may be demonstrated directly in vitro by drug-induced relaxation of precontracted airways (Barns et al. 1988). Bronchodilators promptly reverse airway obstruction in asthmatics. This action believed to be mediated by a direct effect on airway smooth muscle. However, additional pharmacologic effects on the other airway cells (such as capillary endothelium to reduce microvascular leakage and mast cells to reduce release of bronchoconstrictor mediators) may contribute to the overall reduction in airway narrowing. Only three types of bronchodilators are in current clinical use: $\beta$-adrenergic agonists, methylxanthines, and anticholinergics.

$\beta$-adrenergic agonists

Epinephrine has been used to treat asthma since the beginning of the 20 th century. $\beta$ Adrenergic agonists are most widely used and effective bronchodilators for the treatment of asthma. Bronchodilation is mediated by $\beta_{2}$ receptors; $\beta_{2}$ selective drugs (Salmeterol and Formoterol) have been developed that have long duration of effect. $\beta$ Adrenergic agonists lead to relaxation of bronchial smooth muscle that promote bronchodilation. Activation of adenylate cyclase increases the concentration of intracellular cyclic adenosine 3', 5'-monophosphaste (cAMP), leading to activation of specific cAMP-dependent protein kinases that cause relaxation. Relaxation may also be due to inhibition of myosin phosphorylation. $\beta$-adrenergic agonists reverse bronchoconsriction irrespective of the contractile agent. $\beta$ adrenergic agonists prevent release of mediators from a number of inflammatory cells in vitro (Church and Hiroi 1987). In addition, $\beta$ adrenergic agonists increase mucus secretion from submucosal glands and ion transport across airway epithelium. These effects enhance mucociliary clearance caused by asthma (Pavia et al. 1980).

The inhaled route of administration is preferable to the oral route because adverse effects caused by systemic action of the drug are less and also because this route may be more effective. The inhaled drug reaches surface cells (e.g., mast cells or epithelial cells), which are less accessible to the orally administered drug.

Metaproterenol, terbutaline, albuternol, formopterol, bitolterol, salmeterol, and pirbuterol are the classic examples of selective $\beta_{2}$-adrenergic agonists.

$\beta$ agonists improve respiratory symptoms and exercise tolerance despite the small improvement in spirometric measurements. The long acting $\beta$-agonists decrease infection exacerbations as an additional potential benefit. Salmeterol has been shown to reduce adherence of bacteria such as $H$. influenza to airway epithelial cells.

$\beta_{2}$ selective agents cause tachycardia and palpitation by reflex cardiac stimulation secondary to peripheral vasodilation. Muscle tremor is caused by stimulation of $\beta_{2}$ adrenergic receptors in skeletal muscle and is the primary adverse effect of albuterol and bitolterol. Transient hypokalemia may be induced by high dose of these agents.

Anticholinergics

Datura plants contain the muscarinic antagonist and were smoked for relief of asthma centuries ago. Now a days, atropine and ipratropium bromide are the most commonly available anticholinergics.

Antimuscarinic agents specifically antagonize muscarinic receptors. They inhibit reflex cholinergic bronchoconstriction and do not significantly block the direct effects of inflammatory mediators such as histamine and leukotrienes on bronchial smooth muscle and vessels. When given by inhalation, anticholinergics produce bronchodilation by competitively inhibiting cholinergic receptors in bronchial 
smooth muscle. This activity blocks acetylcholine with the net effect being a reduction in cyclic guanosine monophosphate (cGMP) that normally acts to constrict bronchial smooth muscle. Anticholinergic drugs usually are less effective as bronchodilators in asthmatic subjects than $\beta$ adrenergic agonists. Nevertheless, they may have an additive effect with $\beta$ adrenergic agonists.

Atropine reduces mucociliary clearance in normal subjects and in patients with asthma and chronic bronchitis, but the quaternary derivative, ipratropium bromide, even when given in high doses, has no such detectable effect either on normal subjects or in patients with airway disease (Pavia et al. 1980).

Ipratropium bromide has been shown to decrease the effectiveness of voluntary cough on clearing mucus from the airways, which may affect its role in the treatment of patients who have excessive mucus production. Ipratropium has a slower onset of action and a more prolonged bronchodilator effect compared with standard $\beta_{2}$-agonists and has been considered to be less suitable for use on an as needed basis for immediate relief of bronchospasm.

The lack of systemic absorption of ipratropium greatly diminishes the anticholinergic side effects such as blurred vision, urinary retention, nausea, and tachycardia associated with atropine. A significant unwanted effect of inhaled ipratropium bromide is dryness of mouth and throat, bitter taste, cough and nausea. Nebulized ipratropium bromide may precipitate glaucoma in elderly patients because of its direct mydriatic effect on the eye. During sleep, ipratropium also has been shown to improve arterial oxygen saturation and sleep quality.

Tiotropium bromide is a long acting quaternary anticholinergic agent. Tiotropium in human lungs shows approximately 10 fold more potency than ipratropium and protects against cholinergic bronchoconstriction for greater than $24 \mathrm{~h}$.

\section{Methylxanthines}

Methylxanthines such as theophylline are related to caffeine and have been used to treat asthma since 1930. The methylxanthines may produce bronchodilation through numerous mechanisms, including,

- inhibition of phosphodiesterase, thereby increasing cAMP levels

- inhibition of calcium ion influx into smooth muscle

- prostaglandin antagonism

- stimulation of endogenous catecholamines

- adenosine receptor antagonism

- Inhibition of release of mediators from mast cells and leukocytes.

Theophylline inhibits release of mediators from mast cells, increases mucocilliary clearance, and prevents the development of micro vascular leakiness, as would an “anti-inflammatory" drug (Persson and Draco 1988). Theophylline also inhibits some functions of T lymphocytes, which may be relevant to control of chronic inflammation of the airway.

For nocturnal asthma, a single dose of slow release theophylline at bedtime often is effective. This has been demonstrated to reduce overnight declines in $\mathrm{FEV}_{1}$ and morning respiratory symptoms. Taken alone it increases exercise tolerance without improving spirometry tests.

Other theophylline salts, such as choline theophyllinate, offer no advantages over theophylline. The ethylenediamine component of aminophylline has been implicated in allergic reactions. Some derivates such as acepiphylline, diprophylline, and proxophylline, are less effective than theophylline (Weinberger 1984). The most common adverse effects are headache, nausea and vomiting, abdominal discomfort, and restlessness.

\section{Anti-inflammatory drugs}

Although the type of inflammatory responses may differ among diseases, inflammation is a common denominator of several lung diseases. Anti-inflammatory drugs suppress the inflammatory response by inhibiting infiltration and activation of inflammatory cells as well as their synthesis or release of mediators or effects of inflammatory mediators themselves.

\section{Corticosteroids}

Since asthma is viewed as a chronic inflammatory disease and inhaled corticosteroids are known to have low toxicity, they may be considered as first line therapy (Barns 1989). Prednisolone and dexamethasone were effective when they were given systematically to treat asthma but they had no anti-asthmatic activity when they were given by inhalation. Other corticosteroids e.g. beclomethasone dipropionate (BDP), betamethasone and budesonide, were effective in treating asthma when given by inhalation. The antiasthmatic potency of an inhaled steroid is approximately proportional to its potency as an anti-inflammatory agent.

Corticosteroids inhibit the release of arachidonic acid metabolites and platelet activating factor (PAF) from lungs and macrophages by enhancing the production of proteins called lipocortin. Thereby they inhibit the formation of prostaglandins and leukotrienes. These effects occur because of ability of steroid-receptor complex to be transported to the nucleus, where it initiates DNA transcription of specific mRNAs. Corticosteroids potentially inhibit the accumulation of neutrophils, inhibit secretion of human pulmonary macrophages of leukotrienes and prostaglan- 
dins, inhibit formation of interleukins (ILs) such as IL-1, IL-2, IL-3 and IL-5, inhibit degranulation and adherence of eosinophils, reduce number of circulating $\mathrm{T}$ lymphocytes and formation of an $\operatorname{IgE}$ binding suppressive factor. Steroids prevent and reverse the increase in vascular permeability due to inflammatory mediators and may therefore lead to resolution of airway edema. Corticosteroids remain the most effective therapy available for asthma but the legitimate fear of their adverse effects makes using them difficult. Steroids potentiate the effects of $\beta$ adrenergic agonists on bronchial smooth muscle (Barns 1989). Methylprednisolone is given intravenously to patients with severe acute asthma. Inhaled steroids have no proven value in the management of acute asthma. Patients with chronic bronchitis occasionally respond to steroids, possibly because some have an element of undiagnosed asthma.

Corticosteroids inhibit release of ACTH and secretion of cortisol by a negative feed back effect on the pituitary gland. Adverse effects of corticosteroids include fluid retention, increased cell mass, increased appetite, weight gain, osteoporosis, capillary fragility, hypertension, peptic ulceration, diabetes, cataract, and psychosis (Dajani et al. 1981).

\section{Anti-leukotrienes}

Leukotrienes possess potent pro-inflammatory actions resulting in increased vascular permeability, mucus secretion and bronchial hyperresponsiveness. They are derived from the 5-lipoxygenase pathways in mast cells, eosinophils and macrophages. Anti-leukotrienes improve lung function and diminish symptoms, exacerbation rate and the need for rescue bronchodilator. These are drugs of choice in case of aspirin induced asthma, in which patients have high $\mathrm{LTE}_{4}$ levels in urine and nasal secretions and even higher after taking aspirin (Christie et al. 1992).

Leukotriene modifiers are drugs that modify the response of these mediators of inflammation by one of the four ways (Drazen 1997).

a) Cysteinyl LT receptor inhibitors

C-LTs promote eosinophil influx, bronchospasm and mucus hypersecretion, all are considered hallmarks of asthma. C-LT receptor inhibitors antagonize or inhibit leukotrienes predominantly $\mathrm{LTD}_{4}$. These agents inhibit phospholipases, prostaglandins, leukotrienes, and IL-1 synthesis. Probilukast and Iralukast belong to this class (Drazen 1997; Floreani and Rennard 1999).

b) 5-lipoxygenase inhibitors

They prevent the formation of leukotrienes by blocking a 5-lipoxygenase pathway in their synthesis. Zileuton, ZD-2138, ABt-761 belongs to this class (Floreani and Rennard 1999). c) 5-lipoxygenase activating protein (FLAP) inhibitors MK-0591 and MK-886 attenuated the early and late asthmatic response following antigen challenge but not the attendant increase in airway responsiveness to spasmogens (Diamant et al. 1995).

d) Leukotrienes receptor antagonists

Montelukast, Zafirlukast, Pranlukast are selective and high affinity $\mathrm{LT}_{1}$ antagonists (Adcock and Matthews 1998).

Zileuton has shown efficacy in exercise-induced asthma, aspirin induced bronchospasm and following chronic administration, an improvement in pulmonary function (FEV1) and a reduction in oral and inhaled corticosteroid use (Tamaoki et al. 1997). Furthermore, in a small study, zileuton attenuated both airway and blood eosinophilia in nocturnal asthmatics (Wenzel et al. 1995).

Zafirlukast has been demonstrated to attenuate the acute airway obstructive response to allergen and exercise challenge and to improve chronic asthma control both objectively (FEV1, nocturnal awakenings, $\beta$-agonist use) and subjectively.

Montelukast has been shown to block the early and late response to allergen challenge following single dosing, to improve FEV1 in both children (6-14 years) and adults and to protect against the development of exercise induced bronchoconstriction in both children and adults. Tolerance to the bronchoprotective effects of montelukast in attenuating exercise-induced bronchospasm does not develop following at least 12 weeks of therapy.

Pranlukast increases FEV1 within $1 \mathrm{~h}$ of dosing, improves patient summary symptom and nighttime asthma scores and reduces the use of rescue bronchodilators. In patients with moderate persistent asthma, it prevents exacerbations of asthma during reduction of high dose inhaled corticosteroids therapy (Tamaoki et al. 1997).

\section{Mediator release inhibitors}

\section{Cromolyn sodium}

Cromolyn Sodium (Sodium cromoglycate) is a derivative of khellin, an Egyptian herbal remedy. Cromolyn inhibited the release of mediators by allergen in passively sensitized animal and human lung preparations (Cox 1967). Cromolyn was classified as mast cell stabilizer. Cromolyn has variable inhibitory actions on other inflammatory cells including macrophages and eosinophils that may participate in allergic inflammation. In vivo cromolyn can block both the early response that may be mediated by mast cells to allergens and the late response and bronchial hyper responsiveness (Cockcroft and Murdock 1987). Cromolyn Sodium is used for prophylactic treatment and consequently needs to be taken regularly. It is the first choice anti- 
inflammatory drug for children because it has few adverse effects (Bernstein 1985). Cromolyn sodium is classified as an antiallergic drug because it appears to have a specific effect on allergy based inflammation. Several other drugs also may be included in this category.

Nedocromil sodium is a new drug used for prophylaxis. It has a similar pharmacologic profile of activity to cromolyn, is more potent in various tests, and may have a longer duration of action. Ketotifen also is described as a drug to be used for prophylaxis against asthma.

Newer targets in asthma therapy

The current pharmacotherapeutic approaches to asthma have several limitations. First, there is no known asthma cure and little evidence that prevention is possible in susceptible persons. Hence, patients continue to be at risk of symptoms and exacerbations. Mortality remains a severe problem. Finally, the medications have adverse effects. There is even some evidence, albeit conflicting, that cataract formation, osteoporosis and growth impairment, as associated with systemic glucocorticoids, may arise from topical steroids, depending on dosages used. New inhalation devices and new generation beta-agonists are available. At the same time, new understanding of the molecular pathology of asthma has identified several novel therapeutic targets. Agents being tested in early phase clinical trials include antagonists of $\operatorname{IgE}$, cytokines, adhesion molecules and transcription factors.

$\mathrm{TXA}_{2}$ inhibitors

$\mathrm{TXA}_{2}$ is a potent bronchoconstrictor, mucus producer and blood vessel permeability inducer and causes airway hyper responsiveness. Serabenast, domitroban and ozagrel are the

Table 1 Bronchodilators

\begin{tabular}{|c|c|c|c|c|}
\hline Sr. No & Name of plant & Part used/extract/fraction & Major chemical constituent(s) & Reference \\
\hline 1. & Adhatoda vasica Nees & Leaves, Roots & Alkaloids & Paliwa et al. 2000 \\
\hline 2. & $\begin{array}{l}\text { Albizzia lebbeck } \\
\quad \text { (Sareesha_rakat) }\end{array}$ & Stem bark/Aqueous & Saponins & Tripathi and Das 1977 \\
\hline 3. & Alstonia scholaris & Leaves/Ethanol & $\begin{array}{l}\text { Ditamine, Echitamine and } \\
\text { Echitenines }\end{array}$ & Channa et al. 2003 \\
\hline 4. & Artemisia caerulescens & Aerial parts/Butanol & Quercetin, isorhamnetin & Moran et al. 1989 \\
\hline 5. & Belamcanda chinensis & Leaves/Ethanol & Tectorigenin & Singh and Agrawal 1990 \\
\hline 6. & Benincasa hispida & Fruits/Methanol & Triterpenes,Glycosides, Sterols & Kumar and Ramu 2002 \\
\hline 7. & Cissampelos sympodialis & Leaves and root bark/Aqueous & $\begin{array}{l}\text { Warifteine, } \alpha \text {-bisbenzylisoquinoline } \\
\text { alkaloid }\end{array}$ & $\begin{array}{l}\text { Thomas et al. 1995, 1997; } \\
\text { Cortes et al. } 1995\end{array}$ \\
\hline 8. & Clerodendron serratum & Stem bark/Aqueous & Phenolic glycoside & $\begin{array}{l}\text { Gupta 1968; Gupta and } \\
\text { Tripathi } 1973\end{array}$ \\
\hline 9. & Coleus forskohlii & Roots & Forskolin (Diterpenoid) & Marone et al. 1987 \\
\hline 10. & Elaeocarpus spharicus & $\begin{array}{l}\text { Fruits/Aqueous, Pet-ether, Benzene, } \\
\text { Acetone and ethanol }\end{array}$ & $\begin{array}{l}\text { Glycoside, Steroids, Alkaloid, } \\
\text { Flavanoids }\end{array}$ & Singh et al. 2000 \\
\hline 11. & Galphimia glauca & Aerial/Alcohol extract/Ethyl-acetate & Tetragalloylquinic acid, Quercetin & Campos et al. 2001 \\
\hline 12. & Gardenia latifolia & Bark & Saponins & Gupta 1974 \\
\hline 13. & Ginko biloba & Leaves & Ginkgolides & Puglisi et al. 1988 \\
\hline 14. & Mikania glomerata & Leaves/Aqueous, hydroalcohol & Coumarin & Soares de Moura et al. 2002 \\
\hline 15. & Lepidium sativum & Seeds/Ethanol fractions & Alkaloids, Flavonoids & Mali et al. 2008 \\
\hline 16. & Ocimum sanctum & Leaves/Ethanol & Myrcenol, Nerol, Eugenol & Singh and Agrawal 1991 \\
\hline 17. & Passiflora incarnata & Leaves/Methanol & Alkaloids & Dhawan et al. 2003 \\
\hline 18. & Pavetta crassipes & Leaves/Aqueous & Flavanoids, tannins, anthraquinones & Amos et al. 1998 \\
\hline 19. & Picrorrhiza kurroa & Roots/ & Androsin & Stuppner et al. 1991 \\
\hline 20. & Sarcostemma brevistigma & Twigs/Alkaloidal fraction & Bregenin & Saraf and Patwardhan 1988b \\
\hline 21. & Tephrosia purpurea & Aerial parts/Ethanol extract & Flavanoids, Tephrosin & Gokhale et al. 2000 \\
\hline 22. & Tylophora indica & Leaves/Alkaloidal fraction & Tylophorine & Nayampalli et al. 1986 \\
\hline 23. & Vitex negundo & Leaves/Ethanol & $\begin{array}{l}\text { Casticin, isoorientin Chrysophenol } \\
\text { D, Luteolin }\end{array}$ & Nair and Saraf 1995 \\
\hline 24. & Rosmarinus officinalis & Shrub/Aqueous & $\begin{array}{l}\text { Caffeic acid (CA) and Rosmarinic } \\
\text { acid }\end{array}$ & Aqel 1991 \\
\hline 25. & Ephedra sinica & Stems & Ephedrine & Akiba et al. 1979 \\
\hline 26. & Gleditsia sinensis Lam. & Leaves/Decoctions & & Dai et al. 2002 \\
\hline
\end{tabular}


examples of these $\mathrm{TXA}_{2}$ synthetase inhibitors. Ozagrel reduced cough sensitivity to capsaicin and bronchoconstriction due to acetaldehyde. TXA 2 antagonists BAYu3405 produced a modest decrease in airways responsiveness to methacholine following 2 weeks treatment in asthmatics.

Tachykinin receptor antagonists

The first nonpeptide tachykinin receptor antagonist was CP-96345, which is a potent $\mathrm{NK}_{1}$ receptor antagonist. $S R$
48968, GR 159897 and $S R 144190$ are selective nonpeptide $\mathrm{NK}_{2}$ receptor antagonists. $S R 142801$ and $S B 223412$ are selective NK-3 receptor antagonists.

Tryptase inhibitors

Tryptase inhibitors inhibit both early and late reactions. $A P C-366$ inhibited antigen induced late phase response and bronchial hyperresponsiveness to carbachol in sheep. Lactoferrin disrupts the quaternary structure

Table 2 Mast cell stabilizers

\begin{tabular}{|c|c|c|c|c|}
\hline Sr.No. & Name of plant & Part used/extract/fraction & Major chemical constituent(s) & Reference \\
\hline 1. & Achyranthes aspera & Aerial parts/Aqeous & Oleanolic acid & Agrawal and Mehta 2005 \\
\hline 2. & Albizzia lebbeck & Stem bark/Aqueous & Saponins & Tripathi et al. 1979 \\
\hline 3. & Allium cepa & Bulbs/Juice & $\alpha$ and $\beta$ unsaturated Thiosulphinates & Johri et al. 1985 \\
\hline 4. & Aquillaria agallocha & Stem/Aqueous extract & Triterpenoids & Kim et al. 1997 \\
\hline 5. & Azadirachta indica & Leaves/Juice & Nimbin, nimbinine, Nimbandiol, quercetin & Acharya et al. 2003 \\
\hline 6. & Bacopa monniera & Leaves/Ethanol & Bacosides, Alkaloids, Glycosides & Samiulla et al. 2001 \\
\hline 7. & Bidens parviflora & Aerial parts & Glycosides & Wang et al. 2001 \\
\hline 8. & Calotropis procera & Latex & $\begin{array}{l}\alpha \text {-amyrin, } \beta \text {-amyrin calotropin } \\
\text { (Triterpenoid) }\end{array}$ & Kumar and Basu 1994 \\
\hline 9. & Cassia alata & Leaves/Ethanol & Anthraquinones, Flavanoids & Palanichamy et al. 1991 \\
\hline 10. & Cassia obtusifolia & Seeds/Glycosidal fraction & Anthraquinones, Betulinic acid & Kitanaka et al. 1998 \\
\hline 11. & Cassia torosa & Seeds & Gentiobiosides & Kanno et al. 1999 \\
\hline 12. & Cedrus deodara & Wood oil & Himacholol & Shinde et al. 1999 \\
\hline 13. & Citrus unshiu & Peels/ & Flavanoids & Kim et al. 1999 \\
\hline 14. & Clerodendron serratum & Bark/Aqueous & Phenolic glycoside & Gupta 1968 \\
\hline 15. & Cnidium monnieri & Fruits/Ethanol & Osthol & Chen et al. 1988 \\
\hline 16. & Coleus forskohlii & Roots & Forskolin (diterpenoid) & Marone et al. 1987 \\
\hline 17. & Crinum glaucum & Leaves/Aqueous & Alkaloids, lycorine, crinamine & Okpo and Adeyemi 2002 \\
\hline 18. & Curcuma longa & Rhizome & Tumerones, curcuminoids & Ammon and Wahl 1991 \\
\hline 19. & Drymaria cordata & Methanol extracts & & Mukherjee et al. 1997 \\
\hline 20. & Elaeocarpus spharicus & $\begin{array}{l}\text { Fruits/Aqueous, Pet-ether, Benzene, } \\
\text { Acetone and ethanol }\end{array}$ & Glycoside, Steroids, Alkaloid, Flavanoids & Singh et al. 2000 \\
\hline 21. & Gleditsia sinensis & Fruits/Ethanol & Saponins & Dai et al. 2002 \\
\hline 22. & Impatiens textori & Flowers/Ethanolic & Apigenin, uteolin, chrysoeriol & Ishiguro et al. 2000 \\
\hline 23. & Inula racemosa & Roots/Alcohol & Inulolide-a new Sesquiterpene lactone & Srivastava et al. 1999 \\
\hline 24. & Magnolia officinalis & Bark/Aqueous & Honokiol, Magnolol & Shin et al. 2001b \\
\hline 25. & Mentha piperita & Leaves & Flavanoidal glycosides & Inoue et al. 2002 \\
\hline 26. & Ocimum sanctum & Leaves/Aqueous & Myrcenol, Nerol, Eugenol & Sen 1993 \\
\hline 27. & Picrorrhiza kurroa & Roots/ & Androsin & Stuppner et al. 1991 \\
\hline 28. & Solanum xanthocarpum & Roots/Alkaloidal fraction & Solasodine & Chitravanshi et al. 1990 \\
\hline 29. & Striga orobanchioids & Aerial parts/Ethanol & & Harish et al. 2001 \\
\hline 30. & Tephrosia purpurea & Aerial parts/Ethanol extract & Flavanoids, Tephrosin & Gokhale et al. 2000 \\
\hline 31. & Terminalia chebula & Fruits/Aqueous & Ellagic acid, Tannins Chebulagic acid & Shin et al. 2001a \\
\hline 32. & Tinospora cordifolia & Stem/Aqueous & Tinosporin & Nayampalli et al. 1986 \\
\hline 33. & Tylophora asthmatica & Leaves/Alkaloidal & Tylophorine & Geetha et al. 1981 \\
\hline 34. & Vitex negundo & Leaves/Ethanol & $\begin{array}{l}\text { Casticin, isoorientin Chrysophenol D, } \\
\text { Luteolin }\end{array}$ & Nair et al. 1994 \\
\hline
\end{tabular}


of tryptase, also attenuates antigen induced late response and bronchial hyperresponsiveness in allergic sheep.

\section{Cytokine inhibitors}

One of the novel approaches for the treatment of asthma is to target cytokines and develop cytokine modulators as drugs. Two humanized anti-IL-5 monoclonal antibodies, Sch-55700 and SB-240563 reduced blood eosinophil count for several weeks and prevented eosinophils recruitment into the airways after allergen challenge in asthmatic patients. IL-5 signaling inhibitor $G C C$ - $A P 0341$ inhibited IL-5 mediated survival of eosinophils. IL-4 receptor antibodies inhibited allergen induced airway hyperresponsiveness, goblet cell metaplasia and pulmonary eosinophilia in a murine model.
Chemokine inhibitors

A variety of chemokines, one of which is the chemoattractant eotaxin, are secreted by inflamed lung tissue thereby attracting eosinophils. Eotaxin receptor blockers are being investigated, as eosinophils are believed to be major contributors to the pulmonary damage seen in asthma. Monoclonal antibody (7B11) for human $\mathrm{CCR}_{3}$ has shown to completely block the binding and signaling of the known $\mathrm{CCR}_{3}$ ligands, thus blocking the chemotactic response of human eosinophils to all chemokines.

Adhesion molecule antagonists

Interactions of eosinophils with intra cellular adhesion molecule-1 (ICAM-1) are thought to be necessary for eosinophils recruitment into airways. Antibodies to ICAM-1

Table 3 Anti-allergics

\begin{tabular}{|c|c|c|c|c|}
\hline Sr. No. & Name of plant & Part used/extract/fraction & Major chemical constituent(s) & Reference \\
\hline 1. & Adhatoda vasica & Leaves/Methanol & Vasicinol, vasicine & $\begin{array}{l}\text { Muller et al. 1993; Kumar } \\
\text { Suresh } 1979\end{array}$ \\
\hline 2. & Albizzia lebbeck & Stem bark/Aqueous & Saponins & $\begin{array}{l}\text { Baruah et al. 1997; Suresh } \\
\text { et al. } 1981\end{array}$ \\
\hline 3. & Alisma orientale & Rhizomes/Aqueous, Methanol & $\begin{array}{l}\text { Alisol B monoacetate, Alismaketones- } \\
\text { B 23-acetate and -C 23-acetate }\end{array}$ & Kubo et al. 1997 \\
\hline 4. & Aquillaria agallocha & Stem/Aqueous extract & Triterpenoids & Kim et al. 1997 \\
\hline 5. & Asiasarum sieboldi & Roots/Methanol & $\begin{array}{l}\text { Methyleugenol, gamma-asarone, } \\
\text { Elemicin, Asarinin }\end{array}$ & Hashimoto et al. 1994 \\
\hline 6. & Camellia sinensis & Leaves & Flavanoids & Suzuki et al. 2000 \\
\hline 7. & Centipeda minima & Aerial parts & $\begin{array}{l}\text { Flavanoids, Pseuodoguainolide, } \\
\text { sesquiterpene lactones }\end{array}$ & Wu et al. 1985 \\
\hline 8. & Citrus unshiu & Peels/ & Flavanoids & Kim et al. 1999 \\
\hline 9. & Cnidium monnieri & Fruits/Ethanol & Osthol & Matsuda et al. 2002 \\
\hline 10. & Crinum glaucum & Leaves/Aqueous & Alkaloids, lycorine, crinamine & Okpo and Adeyemi 2002 \\
\hline 11. & Curcuma longa & Rhizomes & Curcumin and tetrahydrocurcumin & Suzuki et al. 2000 \\
\hline 12. & Dalbergia odorifera & Heart Wood & Flavanoids, Tannins & Chan et al. 1998 \\
\hline 13. & Desmodium adscendins & Aqueous & Triterpenoid Saponin & Addy 1989 \\
\hline 14. & Galphimia glauca & $\begin{array}{l}\text { Aerial/Alcohol extract/ } \\
\text { Ethyl-acetate }\end{array}$ & Tetragalloylquinic acid, Quercetin & Neszmelyi et al. 1993 \\
\hline 15. & Ginko biloba & Leaves & Ginkgolides & Touvay et al. 1985 \\
\hline 16. & Gleditsia sinensis & Fruits/Ethanol & Saponins & Dai et al. 2002 \\
\hline 17. & Hydrangea macrophylla & Leaves & Glycosides & Matsuda et al. 1999 \\
\hline 18. & Inula racemosa & Roots/Alcohol & $\begin{array}{l}\text { Inulolide-a new Sesquiterpene } \\
\text { lactone }\end{array}$ & Srivastava et al. 1999 \\
\hline 19. & Magnolia officinalis & Bark/Aqueous & Honokiol, Magnolol & Shin et al. 2001b \\
\hline 20. & Sarcostemma brevistigma & Twigs/Alkaloidal fraction & Bregenin & $\begin{array}{l}\text { Saraf and Patwardhan } \\
\text { 1988a }\end{array}$ \\
\hline 21. & Solanum xanthocarpum & Roots/Alkaloidal fraction & Solasodine & Chitravanshi et al. 1990 \\
\hline 22. & Terminalia chebula & Fruits/Aqueous & Ellagic acid, Tannins Chebulagic acid & Shin et al. 2001a \\
\hline 23. & Vitex negundo & Leaves/Ethanol & $\begin{array}{l}\text { Casticin, isoorientin Chrysophenol D, } \\
\text { Luteolin }\end{array}$ & Nair and Saraf 1995 \\
\hline
\end{tabular}


Table 4 Anti-inflammatory agents

\begin{tabular}{|c|c|c|c|c|}
\hline Sr.No. & Name of plant & Part used/extract/fraction & Major chemical constituent(s) & Reference \\
\hline 1. & Asystasia gangetica & Leaves/Methanol, Ethyl Acetate & Isoflavone glycoside, dalhorinin & Akah et al. 2003 \\
\hline 2. & $\begin{array}{l}\text { Aloe vera Tourn.ex Linn. } \\
\text { (Liliaceae) }\end{array}$ & $\begin{array}{l}\text { Leaves/Aqueous, Chloroform and } \\
\text { ethanol }\end{array}$ & $\begin{array}{l}\text { Anthraquinones, sterols, saponins } \\
\text { and carbohydrate }\end{array}$ & Vazquez et al. 1996 \\
\hline 3. & Bryonia laciniosa & Leaves/chloroform extract & Flavonoids & Gupta et al. 2003 \\
\hline 4. & Calotropis procera & Latex & $\begin{array}{l}\alpha \text {-amyrin, } \beta \text {-amyrin calotropin } \\
\text { (Triterpenoid) }\end{array}$ & Kumar and Basu 1994 \\
\hline 5. & Cinnamonun Zeylanicum & oil & $\begin{array}{l}\text { Eugenol, cinnamic aldehyde and } \\
\alpha \text {-terpeniol. }\end{array}$ & \\
\hline 6. & Curcuma longa & Rhizomes & Tumerones, curcuminoids & Ammon and Wahl 1991 \\
\hline 7. & Dalbergia odorifera & Heart Wood & Flavanoids, Tannins & Chan et al. 1998 \\
\hline 8. & Elaeocarpus spharicus & $\begin{array}{l}\text { Fruits/Aqueous, Pet-ether, } \\
\text { Benzene, Acetone and ethanol }\end{array}$ & $\begin{array}{l}\text { Glycoside, Steroids, Alkaloid, } \\
\text { Flavanoids }\end{array}$ & Singh et al. 2000 \\
\hline 10. & Nelsonia canescens & Leaf/ethanol extract & Flavonoids & Owoyele et al. 2005 \\
\hline 11. & Indigofera tinctoria & Whole plant/methanol & Polyphenols & Oli et al. 2005 \\
\hline 12. & Butea frondosa Koen. & Leaves/Aqueous & $\begin{array}{l}\text { Flavonoid, glycosides, proteins and } \\
\text { amino acids. }\end{array}$ & Mengi and Deshpande 1999 \\
\hline 13. & Ocimum sanctum & Leaves/Aqueous & Myrcenol, Nerol, Eugenol & Singh and Agrawal 1991 \\
\hline 14. & Ophiopogon japonicus & Root/aquoes extract & Ruscogenin and ophiopogonin D & Kou et al. 2005 \\
\hline 15. & Pavetta crassipes & Leaves/Aqueous & $\begin{array}{l}\text { Flavanoids, tannins, } \\
\text { anthraquinones }\end{array}$ & Amos et al. 1998 \\
\hline 16. & Tylophora asthmatica & Leaves/Alkaloidal & Tylophorine & Manez et al. 1990 \\
\hline
\end{tabular}

Table 5 Anti-spasmodic agents

\begin{tabular}{|c|c|c|c|c|}
\hline Sr. No. & Name of plant & Part used/extract/fraction & Major chemical constituent(s) & Reference \\
\hline 1. & Aegle marmelos & Leaves/Ethanol & Aegelin, Aegelemine,Aegeline & Arul et al. 2004 \\
\hline 2. & Asiasarum sieboldi & Roots/Methanol & $\begin{array}{l}\text { Methyleugenol, gamma-asarone, Elemicin, } \\
\text { Asarinin }\end{array}$ & Hashimoto et al. 1994 \\
\hline 3. & Asystasia gangetica & Leaves/Methanol, Ethyl acetate & Isoflavone glycoside, dalhorinin & Akah et al. 2003 \\
\hline 4. & Bacopa monniera & Leaves/Ethanolic & Bacosides, Alkaloids, Glycosides & $\begin{array}{l}\text { Dar and Channa 1997; } \\
\text { Channa et al. } 2003\end{array}$ \\
\hline 5. & Belamcanda chinensis & Leaves/Ethanol & Tectorigenin & Singh and Agrawal 1990 \\
\hline 6. & Cissampelos glaberrina & Leaves, Root Bark/Aqueous & Warifteine, $\alpha$-bisbenzylisoquinoline alkaloid & $\begin{array}{l}\text { Thomas et al. 1995; Cortes } \\
\text { et al. } 1995\end{array}$ \\
\hline 7. & Clerodendron serratum & Stem bark/Aqueous & Phenolic glycoside & Gupta 1968 \\
\hline 8. & Cnidium monnieri & Fruits/Ethanol & Osthol & Chen et al. 1988 \\
\hline 9. & Coleus forskohlii & Roots & Forskolin (diterpenoid) & Marone et al. 1987 \\
\hline 10. & Crinum glaucum & Leaves/Aqueous & Alkaloids, lycorine, crinamine & Okpo and Adeyemi 2002 \\
\hline 11. & Drymis winteri & Bark & Terpene & Sayah et al. 1998 \\
\hline 12. & Ferula ovina & Aerial parts/Ethanol & $\begin{array}{l}\text { Carvacrol, alpha-pinene, geranyl isovalerate } \\
\text { and geranyl propionate }\end{array}$ & Khalil et al. 1990 \\
\hline 13. & Ferula sinica & Roots/Ethanol & Resins & Aqel et al. 1991 \\
\hline 14. & Pavetta crassipes & Leaves/Aqueous & Flavanoids, tannins, anthraquinones & Amos et al. 1998 \\
\hline 15. & Saussurea leppa & Alkaloidal fraction & Sesquiterpene lactone, Terpenoids & Dutta et al. 1968 \\
\hline 16. & Striga orobanchioids & Aerial parts/Ethanol & $? ?$ & Harish et al. 2001 \\
\hline 17. & Thymus vulgaris & Ethanol & Flavanones & Meister et al. 1999 \\
\hline 18. & Tylophora asthmatica & Leaves/Alkaloidal & Tylophorine & $\begin{array}{l}\text { Haranath and } \\
\text { Shyamalakumari 1975; } \\
\text { Udapa et al. } 1991\end{array}$ \\
\hline
\end{tabular}


blocked both eosinophils recruitment into the airways in the monkey model of asthma and importantly the increase in airway reactivity associated with allergen challenge

\section{Phosphodiesterase inhibitors}

Considerable interest has been generated in the potential utility of isoenzyme-selective inhibitors of cyclic nucleotide Phosphodiesterase (PDE) in the treatment of asthma and other inflammatory disorders. The scientific foundation for this interest is based upon two fundamental principles. First, inhibition of PDE activity increases the cellular content of two key second messengers, cAMP and cGMP, thereby activating specific protein phosphorylation cascades that elicit a variety of functional responses. Increases in cAMP content suppress a broad array of functions in inflammatory and immune cells. Both cAMP and cGMP mediate bronchodilation. PDE3 inhibitor enoxamine was shown to decrease lung resistance and increase compliance in patients with decompensated chronic pulmonary disease. Benzafentrine administered to normal volunteers by inhalation produced bronchodilation. Zaprinast is PDE5 inhibitor; it reduced exercise-induced bronchoconstriction but not histamine-induced bronchoconstriction. Most of the work now is focused on selectively targeting PDE4, primarily because inhibitors of this isoenzyme family have a notably appealing therapeutic profile; broad-spectrum anti-inflammatory activity coupled with additional bronchodilatory and neuromodulatory action. Rolipram, $L A S$ 31025, RP-73401 and denbufylline are selective $\mathrm{PDE}_{4}$ inhibitors. $S B$ 207499, V11294A, CP-220 and roflumilast are $\mathrm{PDE}_{4}$ inhibitors with less gastrointestinal side effects.

\section{Endothelin modulators}

There are two approaches for ET-1 directed therapeutics- (1) Inhibitors of endothelin-converting enzyme (ECE), which mediates the synthesis of ET-1 from its precursor; (2) Receptor antagonists of the effects of ET-1 at the end organ level. These agents reverse and/or prevent the increase in pulmonary artery pressure and vascular remodeling elicited by acute or chronic hypoxia. Examples are BQ-123, SB217242 and bosentan.

\section{Herbal drugs in bronchial asthma}

Many Ayurvedic plants have been described to be useful in the treatment of various bronchial disorders including

Table 6 Miscellaneous agents

\begin{tabular}{|c|c|c|c|c|}
\hline Sr No & Name of plant & Part used/extract/fraction & Major chemical constituent(s) & Reference \\
\hline & Lipoxygenase inhibitors & & & \\
\hline 1. & Allium cepa & Bulbs/Juice & $\alpha$ and $\beta$ unsaturated Thiosulphinates & Bayer et al. 1989 \\
\hline 2. & Boswellia serrata & Gum resin/Ethanol & Bosewellic acid & Ammon et al. 1991 \\
\hline 3. & Coleus forskohlii & Roots & Forskolin (diterpenoid) & Marone et al. 1987 \\
\hline \multirow[t]{2}{*}{4.} & Proustia pyrifolia & Whole plant/methanol & B-sitosterol, quercetin and dihydroquercetin & Delporte et al. 2005 \\
\hline & Platelet Activating Factor ( & AF) inhibitors & & \\
\hline 1. & Allium сера & Bulbs/Juice & $\alpha$ and $\beta$ unsaturated Thiosulphinates & Dorsch et al. 1987 \\
\hline 2. & Galphimia glauca & $\begin{array}{l}\text { Aerial/Alcohol extract/ } \\
\text { Ethyl-acetate }\end{array}$ & Tetragalloylquinic acid, Quercetin & Neszmelyi et al. 1993 \\
\hline 3. & Impatiens textori & Flowers/Ethanol & Apigenin, uteolin, chrysoeriol & Ueda et al. 2003 \\
\hline \multirow[t]{2}{*}{4.} & Picrorrhiza kurroa & Roots/ & Androsin & Stuppner et al. 1991 \\
\hline & Cyclooxygenase inhibitor & & & \\
\hline 1. & Allium сера & Bulbs/Juice & $\alpha$ and $\beta$ unsaturated Thiosulphinates & Bayer et al. 1989 \\
\hline 2. & Magnolia obovate & Stem bark & Magnolol and honokiol & Lee et al. 2005 \\
\hline \multirow[t]{2}{*}{3.} & Crataegus pionatifida & Fruit & Flavanoids & Kao et al. 2005 \\
\hline & Interleukins (Ils) biosynthe & s inhibitors & & \\
\hline 1. & Calocedrus formosana & Bark/alcohol & Sugiol & Chao et al. 2005 \\
\hline 2. & Nidularium procerum & Leaves/Aqeous extract & & $\begin{array}{l}\text { Vieira-de-Abreu et al. } \\
2005\end{array}$ \\
\hline 3. & Walthenia indica & Whole plant/ & flavanoids & Rao et al. 2005 \\
\hline \multirow[t]{2}{*}{4.} & $\begin{array}{l}\text { Mahonia aquifolium Nutt. } \\
\text { Berberidaceae }\end{array}$ & Stem bark/hydroalcohol extract & $\begin{array}{l}\text { Polysaccharides Protoberberine and } \\
\text { bisbenzylisoquinoline (BBI) alkaloids } \\
\text { berbamine, tetrandrine }\end{array}$ & Kostalova et al. 2001 \\
\hline & Leukotriene biosynthesis in & ibitors & & \\
\hline 1. & Nigella sativa & Seeds oil & Thymoquinone (TQ) & El Gazzar et al. 2006 \\
\hline
\end{tabular}


Table 7 Antianaphylactic drugs

\begin{tabular}{|c|c|c|c|c|}
\hline Sr. No. & Name of plant & Part used/extract/fraction & Major chemical constituent(s) & Reference \\
\hline 1. & Xanthii fructus & Whole plant/Aquous & $\begin{array}{l}\text { Saponin, flavones, Caffeic acid, 1,4- } \\
\text { dicaffeoylquinic acid, sesquiterpene } \\
\text { lactones }\end{array}$ & Hong et al. 2003 \\
\hline 2. & Lycopus lucidus & Whole plant/Aqueous & Betulinic acid, pentacyclic Triterpenes & Shin et al. 2005 \\
\hline 3. & Poncirus trifoliata & Fruit/Aqueous & Flavonoids & Kim et al. 1999 \\
\hline 4. & Trichopus zeylanicus & Leaves/butanol & Lipoprotein/Glycolipoprotein & Subramoniam et al. 1999 \\
\hline 5. & Cryptotympana atrata & Whole plant/Aquous & oleanolic acid & Shin et al. 1999 \\
\hline 6. & Striga orobanchioides & Whole plant/Aquous, ethanolic & Flavonoids, apigenin and luteolin & Harish et al. 2001 \\
\hline 7. & Crinum glaucum & Bulbs/Aqueous & Alkaloids & Okpo and Adeyemi 2002 \\
\hline 8. & Acanthopanax senticosus & Stem/Aqueous & $\begin{array}{l}\text { Acanthoside A, B \& C, Chiisanoside, } \\
\text { Senticoside, Saponin, flavones, }\end{array}$ & Yi et al. 2002 \\
\hline 9. & Syzygium aromaticum & Flower bud/Aqueous & Phenols & Kim et al. 1998 \\
\hline 10 & Terminalia chebula & Fruit/Aqueous & Tannins & Shin et al. 2001a \\
\hline 11 & Vitex rotundifolia & Fruit/Aqueous & Flavonoids & Shin et al. 2000 \\
\hline
\end{tabular}

bronchial asthma (Kumar Suresh 1979). The use of medicinal plants and natural products increased dramatically in the last two decades in all over the world. More than 400 medicinal plant species have been used ethanopharmacologically and traditionally to treat the symptoms of asthmatic and allergic disorders worldwide.

Classification of anti asthmatic herbs based on mechanism of action

Some herbal alternatives employed in asthma are proven to provide symptomatic relief and assist in the inhibition of disease development as well. These herbs therefore have multifaceted roles to play in the management of asthma suggesting different sites of action within the body. Based on the possible mechanism of action reported, plant antiasthmatics may be classified as shown in tables (Tables 1, 2, $3,4,5,6,7$ and 8 ).

\section{Conclusion}

Herbal approaches have regained their popularity, with their efficacy and safety aspects being supported by controlled clinical studies. The herbal approaches have offered effective mast cell stabilizers like sodium cromolyn and sodium cromoglycate developed from khellin and antileukotriene products like-boswellic acids. Ongoing re-

Table 8 Immunomodulatory drugs

\begin{tabular}{|c|c|c|c|c|}
\hline Sr. No. & Name of plant & Part used/extract/fraction & Major chemical constituent(s) & Reference \\
\hline 1. & Picrorhiza scrophulariiflora & $\begin{array}{l}\text { Rhizomes/Pet. ether, Diethyl ether } \\
\text { and methanol }\end{array}$ & Apocynin, androsin and picroside II & Smit et al. 2000 \\
\hline 2. & Trichilia glabra & Leaf/Aqueous & Polysaccharides & Benancia et al. 2000 \\
\hline 3. & Cedrela tubiflora & Leaf/Aqueous & Gallic acid, polysaccharides & Benancia et al. 1995 \\
\hline 4. & Ipomoea carnea & Leaf/Aqueous & Nortropane alkaloids, calystegines $\beta_{2}$ & Hueza et al. 2003 \\
\hline 5. & Withania somnifera & Coded extracts & & $\begin{array}{l}\text { Rasool and Varalakshmi } \\
2006\end{array}$ \\
\hline 6. & Clausena excauata & Wood/Aqueous & $\begin{array}{l}\text { Phenolic compounds, } \\
\text { furanocoumarins, flavanoids and } \\
\text { carbazole alkaloid }\end{array}$ & Manosroi et al. 2005 \\
\hline 7. & Magnifera indica & Bark/Alcohol, ether & Magniferin & Makare et al. 2001 \\
\hline 8. & Cleome viscosa & Aerial parts/Aqueous, ethanolic & Alkaloids, saponins & Tiwari et al. 2003 \\
\hline 9. & Plantago ovata & Seeds/Aqueous & Polysaccharides glycosides & Rezaeipoor et al. 2000 \\
\hline 10. & Typhae angustifolia & Pollen/ethanol & $\begin{array}{l}\text { Phenolic compounds, } \\
\text { flavonesTriterpenes And } \beta \text {-sitosterol }\end{array}$ & Qin and Sun 2005 \\
\hline 11. & Angelica sinensis & Roots/Aqueous and ethanolic & Polysaccharides & Yang et al. 2006 \\
\hline 12. & Boerhaavia diffusa & Roots/ethanol & Alkaloids & Mungantiwar et al. 1999 \\
\hline 13. & Tephrosia purpurea & Aerial parts/Ethanol & Flavanoids & Damre et al. 2003 \\
\hline
\end{tabular}


search worldwide has provided valuable clues regarding the precise mechanism of action of these herbal alternatives and these herbs, have shown interesting results in various target specific biological activities such as bronchodilation, mast cell stabilization, anti-anaphylactic, anti-inflammatory, antispasmodic, anti-allergic, immunomodulatory and inhibition of mediators viz., leukotrienes, lipoxygenase, cyclooxygenase, platelet activating, phosphodiesterase and cytokine, in the treatment of asthma.

Some herbal alternatives employed in these traditions are proven to provide symptomatic relief and assist in the inhibition of disease development as well. In nutshell, attempt should be made to develop polyherbal formulations which contain various herbs acting at particular sites of the pathophysiological cascade of asthma for prophylaxis as well as for the treatment of asthma and subsequent clinical studies on them.

\section{References}

Acharya SB, Yanpallewar SU, Singh RK (2003) A preliminary study on the effect of Azadiracchta indica on bronchial smooth muscles and mast cells. J Nat Rem 3:78-82

Adcock IM, Matthews JC (1998) New drugs for asthma. Drug Discov Today 3:395-419

Addy ME (1989) Several chromatographically distinctive fractions of Desmodium adscendens inhibit smooth muscle contractions. Int $\mathbf{J}$ Crude Drug Res 27:81-91

Agrawal BB, Mehta AA (2005) Phyto-pharmacological investigation of Moringa oleifera and Achyranthus aspera for their antiasthmatic activity. Ph.D. thesis, Gujarat University

Akah PA, Ezike AC, Nwafor SV, Okoli CO, Enwerem NM (2003) Evaluation of the anti-asthmatic property of Asystasia gangetica leaf extracts. J Ethnopharmacol 89:25-36

Akiba K, Onodera K, Kisara K, Fujikura H (1979) Interaction of dpseudoephidrine with water soluble extracts of Platycodi radix on acute toxicity. Nippon Yakurigaku Zasshi 75:201-206

Ammon HP, Mack T, Singh GB, Safayhi H (1991) Inhibition of leukotriene $\mathrm{B}_{4}$ formation in rat peritoneal neutrophils by an ethanolic extract of the gum resin exudates of Boswellia serrata. Planta Med 57:203-207

Ammon HP, Wahl MA (1991) Pharmacology of Curcuma longa. Planta Med 57:1-7

Amos S, Gamaniel K, Akah P, Wambebe C (1998) Anti-inflammatory and muscle relaxant effect of aqueous extract of Pavetta crassipes leaves. Fitoterapia 69:425-429

Aqel MB (1991) Relaxant effect of the volatile oil of Romarinus officinalis on tracheal smooth muscle. J Ethnopharmacol 33:57-62

Aqel MB, al-Khalil S, Afifi F, al-Eisawi D (1991) Relaxant effects of Ferula sinaica root extract on rabbit and guinea pig smooth muscle. J Ethnopharmacol 31:373-381

Arul V, Miyazaki S, Dhananjayan R (2004) Mechanisms of the contractile effect of the alcoholic extract of Aegle marmelos Corr on isolated guinea pig ileum and tracheal chain. Phytomedicine $11: 679-683$

Barns PJ (1989) New concept in the pathogenesis of bronchial hyperresponsiveness and asthma. J Allergy Clin Immunol 83:1013-1026

Barns PJ, Chung KF, Page CP (1988) Inflammatory mediators and asthma. Pharmacol Rev 40:49-84
Baruah CC, Gupta PP, Patnaik GK, Nath A, Kulshreshtha DK, Dhawan BN (1997) Anti-allergic and mast cell stabilizing activity of Albizzia lebbeck. Ind Veterinary Med J 21:127-132

Bayer T, Breu W, Seligmann O, Wray V, Wagner H (1989) Biologically active thiosulphinates and $\alpha$-sulphinyl disulphides from Allium cepa. Phytochemistry 28:2373-2377

Benancia F, Courreges MC, Coulombie FC (2000) In vivo and in vitro immunomodulatory activities of Trichilia glabra aqueous leaf extracts. J Ethnopharmacol 69:199-205

Benancia F, Courreges MC, Nores MM, Coulombie FC (1995) Immunomodulatory activities of Cedrela tubiflora leaf aqueous extracts. J Ethnopharmacol 49:133-139

Bernstein IL (1985) Cromolyn sodium in the treatment of asthma: coming of age in the United States. J Aller Clin Immunol 76:381-388

Campos MG, Toxqui E, Tortoriello J, Oropeza MV, Ponce H, Vargas MH, Montano LM (2001) Galphimia glauca organic fraction antagonized LTD (4)-induced contraction in guinea pig airways. J Ethnopharmacol 74:7-15

Chan SC, Chang YS, Wang JP, Chen SC, Kuo SC (1998) Three new flavonoids and anti-allergic, anti-inflammatory constituents from the heartwood of Dalbergia odorifera. Planta Med 64:153-158

Channa S, Dar A, Yaqoob M, Anjum S, Sultani Z, Atta-ur-Rahman (2003) Broncho-vasodilatory activity of fractions and pure constituents isolated from Bacopa monniera. J Ethnopharmacol 86:27-35

Chao KP, Hua KF, Hsu HY, Su YC, Chang ST (2005) Antiinflammatory activity of sugiol, a diterpene isolated from Calocedrus formosana bark. Planta Med 71:300-305

Chen ZC, Duan XB, Liu KR (1988) The anti allergic activity of osthol extracted from the fruits of Cnidiun monnieri (L.) Cusson. Acta Pharmaceutica Sinica 23:96-99

Chitravanshi VC, Gupta PP, Kulshrestha DK, Kar K, Dhawan BN (1990) Anti-allergic activity of Solanum xanthocarpum. Ind J Pharmacol 22:23-24

Christie PE, Tagari P, Ford-Hutchinson AW, Black C et al (1992) Urinary leukotriene $\mathrm{E}_{4}$ after lysine -aspirin inhalation in asthmatic subjects. Am Rev Respir Dis 146:1531-1534

Church MM, Hiroi J (1987) Inhibition of IgE-dependant histamine release from human dispersed lung mast cells by anti-allergic drugs and salbutamol. Br J Pharmacol 90:421-429

Cockcroft DW, Murdock KY (1987) Comparative effects of inhaled salbutamol, sodium chromoglycate and beclomethasone dipropionate on allergen-induced early asthmatic responses, late asthmatic responses and increased bronchial responsiveness to histamine. $\mathrm{J}$ Aller Clin Immunol 79:734-740

Cortes SF, Alencar JI, Thomas G, Filho JMB (1995) Spasmolytic action of warifteine, a bisbenzylisoquinoline alkaloid isolated from the root bark of Cissampelos sympodialis Eichl. Phytother Res 9:579-583

Cox JSG (1967) Disodium chromoglycate (FPL 670) (Intal): a specific inhibitor of reaginic antibody-antigen mechanisms. Nature 216:1328-1329

Dai Y, Chan YP, Chu LM, Bu PP (2002) Antiallergic and antiinflammatory properties of the ethanolic extract from Gleditsia sinensis. Biol Pharm Bull 5:1179-1182

Dajani BM, Sliman NA, Shubair KS, Hamzeh YS (1981) Bronchospasm caused by intravenous hydrocortisone sodium succinate (Solu-Cortef) in aspirin sensitive asthmatics. J Allergy Clin Immunol 68:201-206

Damre AS, Gokhale AB, Phadke AS, Kulkarni KR, Saraf MN (2003) Studies on the immunomodulatory activity of flavonoidal fraction of Tephrosia purpurea. Fitoterapia 74:257-261

Dar A, Channa S (1997) Relaxant effect of ethanol extract of Bacopa monniera on trachea, pulmonary and aorta from rabbit and guinea pig. Phytother Res 11:323-325

Delporte C, Backhouse N, Erazo S, Negrete R, Vidal P, Silva X, Lopez-Perez JL, Feliciano AS, Munoz O (2005) Analgesic- 
antiinflammatory properties of Proustia pyrifolia. J Ethnopharmacol 99:119-124

Dhawan K, Kumar S, Sharma A (2003) Anti-asthmatic activity of the methanol extract of leaves of Passiflora incarnata. Phytother Res $17: 821-822$

Diamant Z, Timmers MC, Vander Veen H, Friedman BS, De Smet M, Depre M, Hilliard D, Bel EH, Sterk PJ (1995) The effect of MK0591, a novel 5-lipoxygenase activating protein inhibitor on leukotriene biosynthesis and allergen-induced airway responses in asthmatic subjects in vivo. J Aller Clin Immunol 95:42-51

Dorsch W, Ettl M, Hein G, Scheftner P, Weber J, Bayer T, Wagner H (1987) Anti-asthmatic effects of onions. Inhibition of platelet activating factor induced bronchial obstruction by onion oils. Int Arch Allergy App Immunol 82:535-536

Drazen JM (1997) Pharmacology of leukotriene receptor antagonists and 5-lipoxygenase inhibitors in the management of asthma. Pharmacotherapy 17:22S-30S

Dutta NK, Sastry M, Tamhane RG (1968) Pharmacological actions of an alkaloidal fraction isolated from Saussurea leppa (Clarke). Curr Sci 37:550-551

El Gazzar M, El Mezayen R, Nicolls MR, Marecki JC, Dreskin SC (2006) Downregulation of leukotriene biosynthesis by thymoquinone attenuates airway inflammation in a mouse model of allergic asthma. Biochim Biophys Acta 1970:1088-1095

Floreani AA, Rennard SI (1999) The role of cigarette smokes in the pathogenesis of asthma and as a trigger for acute symptoms. Curr Opinion Pulm Med 5:38-46

Geetha VS, Viswanathan S, Kameswaran L (1981) Comparision of total alkaloids of Tylophora indica and disodium cromoglycate on mast cell stabilization. Ind J Pharmacol 13:199-201

Gokhale AB, Dikshit VJ, Damre AS, Kulkarni KR, Saraf MN (2000) Influence of ethanolic extract of Tephrosia purpurea Linn. on mast cells and erythrocytes membrane integrity. Ind J Exp Biol $38: 837-840$

Goyal BR, Agrawal BB, Goyal RK, Mehta AA (2007) Pharmacological classification of herbal anti-asthmatics. Orient Pharm Exp Med 7:11-25

Gupta M, Mazumdar UK, Sivakumar T, Vamsi ML, Karki S, Sambathkumar R, Manikandan L (2003) Evaluation of antiinflammatory activity of chloroform extract of Bryonia laciniosa in experimental animal models. Biol Pharm Bull 26:1342-1344

Gupta SS (1968) Development of anti-histaminic and anti-allergic activity after prolonged administration of a plant saponin from Clerodendron serraum. J Pharm Phramacol 20:801-802

Gupta SS (1974) Some observations on the anti-asthmatic effect of the saponins of Gardenia latifolia. Aspect Aller Appl Immunol 7:198-204

Gupta SS, Tripathi RM (1973) Effect of chronic treatment of the saponin of Clerodendron serratum on disruption of mesenteric mast cells of rats. Aspect Aller Appl Immunol 6:177-188

Haranath PSRK, Shyamalakumari S (1975) Experimental study on the mode of action of Tylophora asthmatica in bronchial asthma. Ind J Med Res 63:661-670

Harish MS, Mallikarjun N, Badami S (2001) Antihistaminic and mast cell-stabilizing activity of Striga orobanchioides. J Ethnopharmacol 76:197-200

Hashimoto K, Yanagisawa T, Okui Y, Ikeya Y, Maruno M, Fujita T (1994) Studies on anti-allergic components in the roots of Asiasarum sieboldi. Planta Med 60:124-127

Hong SH, Jeong HJ, Kim HM (2003) Inhibitory effects of Xanthii fructus extract on mast cell mediated allergic reactions in murine model. J Ethnopharmacol 88:229-234

Hueza IM, Fonseca ESM, Paulino CA, Haraguchi M, Gorniak SL (2003) Evaluation of immunomodulatory activity of Ipomoea carnea on peritoneal cells of rats. J Ethnopharmacol 87:181186
Huntley A, Ernst E (2000) Herbal medicines for asthma: a systematic review. Thorax 55:925-29

Inoue T, Sugimoto Y, Masuda H, Kamei C (2002) Antiallergic effect of flavonoids obtained from Mentha piperita L. Biol Pharm Bull 25:256-259

Ishiguro K, Ueda Y, Iwaoka E, Oku H (2000) Antiallergic and antipruritic effect of Impatiens textori. Phytomedicine 7:94-97

Johri RK, Zutshi U, Kameshwaran L, Atal CK (1985) Effect of quercetin and Albizzia saponins on rat mast cell. Ind J Physiol Pharmacol 29:43-46

Kanno M, Shibano T, Takido M, Kitanaka S (1999) Anti-allergic agent from natural sources.2.structures and leukotriene releaseinhibitory effect of torososide $\mathrm{B}$ and torosachrysone 8-O-6malonyl beta gentiobioside from Cassia torosa Cav. Chem Pharm Bull 47:915-918

Kao ES, Wang CJ, Lin WL, Yin YF, Wang CP, Tseng TH (2005) Antiinflammatory potential of flavonoids contents from dried fruits of Crataegus pinnatifida in vitro and in vivo. J Agric Food Chem 53:430-436

Khalil SA, Aqel M, Afifi F, Eisawi DA (1990) Effect of an aqueous extract of Ferula ovina on rabbit and guinea pig smooth muscle. J Ethnopharmacol 30:35-42

Kim DK, Lee KT, Eun JS, Zee OP, Lim JP, Eum SS, Kim SH, Shin TY (1999) Anti-allergic components from peels of Citrus unshiu. Arch Pharm Res 22:642-645

Kim HM, Lee EH, Hong SH, Song HJ, Shin MK, Kim SH, Shin TY (1998) Effect of Syzygium aromaticum extract on immediate hypersensitivity in rats. J Ethnopharmacol 60:125-131

Kim YC, Lee EH, Lee YM, Kim HK, Song BK, Lee EJ, Kim HM (1997) Effect of the aqueous extract of Aquillaria agallocha stem on the immediate hypersensitivity reactions. J Ethnopharmacol $58: 31-38$

Kitanaka S, Nakayama T, Shibano T, Ohkoshi E, Takido M (1998) Anti-allergic agent from natural sources, structures and inhibitory effect of histamine release of naphthopyrone glycosides from seeds of Cassia obtusifolia L. Chem Pharm Bull 46:1650-1652

Kostalova D, Bukovsky M, Koscova H, Kardosova A (2001) Anticompliment activity of Mahonia aquigolium bisbenzylisoquinoline alkaloids and berberine extract. Ceska Slov Farm 50:286-289

Kou J, Sun Y, Lin Y, Cheng Z, Zheng W, Yu B, Xu Q (2005) Antiinflammatory activities of aqueous extract from radix Ophiopogon japonicus and its two constituents. Biol Pharm Bull 28:1234-1238

Kubo M, Matsuda H, Tomohiro N, Yoshikawa M (1997) Studies on Alismatis rhizome; Anti-allergic effects of methanol extract and six terpene components from Alismatis rhizoma (Dried rhizome of Alisma orientale). Biol Pharm Bull 20:511-516

Kumar DA, Ramu P (2002) Effect of methanolic extract of Benincasa hispida against histamine and acetylcholine induced bronchospasm in guinea pigs. Ind J Pharmacol 34:365-366

Kumar Suresh (1979) Scientific Appraisal of Adhatoda vasica_Nees (Vasaka) J NIMA XXIII: 257-261

Kumar VL, Basu N (1994) Anti-inflammatory activity of the latex of Calotropis procera. J Ethnopharmacol 44:123-125

Lee J, Jung E, Park J, Jung K, Lee S, Hong S, Park J, Park E, Kim J, Park S, Park D (2005) Antiinflammatory effects of magnolol and honokiol are mediated through inhibition of the downstream pathway of MEKK-1 in NR-kB activation signaling. Planta Med: 71:338-343

Makare N, Bodhankar S, Rangari V (2001) Immunomodulatory activity of alcoholic extract of Mangifera indica L. in mice. J Ethnopharmacol 78:133-137

Mali RG, Mahajan SG, Mehta AA (2008) Studies on Bronchodilatory effect of Lepidium sativum against allergen induced bronchospasm in Guinea pigs. Pharmacog Mag 4:189-192 
Manez S, Alcaraz MJ, Paya M, Rios JL, Hancke JL (1990) Selected extracts from medicinal plants as anti-inflammatory agents. Planta Med 56:656

Manosroi A, Saraphanchotiwitthaya A, Manosroi J (2005) In vivo immunomodulating activity of wood extracts from Clausena excavate Burm.F. J Ethnopharmacol 102:5-9

Marone G, Columbo M, Triggiani M, Cirillo R, Genovese A, Formisano S (1987) Inhibition of IgE mediated release of histamine and peptide leukotriene from human basophils and mast cells by forskolin. Biochem Pharmacol 36:13-20

Matsuda H, Shimoda H, Yamahara J, Yoshikawa M (1999) Effect of phyllodulcin, hydrangenol, and their 8-O-glucosides, and thunberginols $\mathrm{A}$ and $\mathrm{F}$ from Hydrangea macrophylla var. thunbergii on passive cutaneous anaphylaxis reaction in rats. Biol Pharm Bull 22:870-872

Matsuda H, Tomohiro N, Ido Y, Kubo M (2002) Anti-allergic effects of Cnidii monnieri (dried fruits of Cnidium monnier) and its major component, osthol. Biol Pharm Bull 25:809-812

Meister A, Bernhard G, Chrisoffel V, Buschauer A (1999) Antispasmodic activity of Thymus vulgaris extract on isolated g.pig trachea: discrimination between drug and ethanol effects. Planta Med 65:512-516

Mengi SA, Deshpande SG (1999) Anti-inflammatory activity of Butea frondosa leaves. Fitoterapia 70:521-522

Moran A, Carron R, Martin ML, San Roman L (1989) Anti-asthmatic activity of Artemisia caerulescens subsp. gallica. Planta Med 55:351-353

Mukherjee PK, Saha K, Bhattacharya S, Giri SN, Pal M, Saha BP (1997) Studies on antitussive activity of Drymaria cordata Willd. J Ethnopharmacol 56:77-80

Muller A, Antus S, Bittinger M, Dorsch W, Kaas A, Kreher B et al (1993) Chemistry and pharmacology of the antiasthmatic plants Galphimia glauca, Adhatoda vasica and Picrorrhiza kurroa. Planta Med 59(A5):86

Mungantiwar AA, Nair AM, Shinde UA, Dikshit VJ, Saraf MN, Thakur VS, Sainis KB (1999) Studies on the immunomodulatory effects of Boerhaavia diffusa alkaloidal fraction. J Ethnopharmacol 65:125-131

Nair AM, Saraf MN (1995) Inhibition of antigen and compound 48/80 induced contractions of guinea pig trachea by the ethanolic extract of the leaves of Vitex negundo Linn. Ind J Pharmacol 27:230-233

Nair AM, Tamhankar CP, Saraf MN (1994) Studies on the mast cell stabilizing activity of Vitex negundo Linn. Ind Drugs 32:277-282

National Institute of Health (1997) Expert Panel Report 2. Guidelines for the diagnosis and Management of asthma. NIH Publication. No.97-4051

Nayampalli S, Desai NK, Ainapure SS (1986) Anti-allergic properties of Tinospora cordifolia in animal models. Ind $\mathrm{J}$ Pharmacol 18:250-252

Neszmelyi A, Kreher B, Muller A, Dorsch W, Wagner H (1993) Tetragalloylquinic acid, the major Antiasthmatic principle of Galphimia glauca. Planta Med 59:164-167

Okpo SO, Adeyemi OO (2002) The anti-allergic effects of Crinum glaucum aqueous extract. Phytomedicine 9:438-441

Oli RG, Manikandan L, Swarna FB, Manikandan P, Khosa RL (2005) Evaluation of antiinflammatory potential of Indigofera tinctoria extract in rats. Ind $\mathrm{J}$ Nat Prod 21:12-15

Owoyele VB, Oloriegbe YY, Balogun EA, Soladoye AO (2005) Analgesic and antiinflammatory properties of Nelsonia canescens leaf extract. J Ethnopharmacol 99:153-156

Palanichamy S, Amala Bhaskar E, Nagarajan S (1991) Effect of Cassia alata leaf extract on mast cell stabilization. Ind $\mathrm{J}$ Pharmacol 23:189-191

Paliwa JK, Dwiwedi AK, Singh S (2000) Pharmacokinetics and insitu absorption tudies of a new anti-allergic compound 73/602 in rats. Int J Pharm 197:213-220
Pavia D, Batement JRM, Clarke SW (1980) Deposition and clearance of inhaled particles. Bull Eur Physiopath Resp 16:335-366

Persson CG, Draco AB (1988) Xanthine as airway anti-inflammatory drugs. J Allergy Clin Immunol 81:615-617

Puglisi L, Salvadori S, Gabrielli G, Pasargiklian R (1988) Pharmacology of natural compounds. Smooth muscle relaxant activity induced by a Ginkgo biloba L. extract on guinea-pig trachea. Pharmacol Res Comm 20:573-589

Qin F, Sun HX (2005) Immunosuppressive activity of pollen Typhae ethanol extract on the immune responses in mice. J Ethnopharmacol 102:424-429

Rao YK, Fang SH, Tzeng YM (2005) Inhibitory effects of the flavonoids isplated from Waltheria indica on the production of NO, TNF-alfa and IL-2 in activated macrophages. Biol Pharm Bull 28:912-915

Rasool M, Varalakshmi P (2006) Immunomodulatory role of Withania somnifera root powder on experimental induced inflammation: An in vivo and in vitro study. Vascul Pharmacol 44:406-410

Rezaeipoor R, Saeidnia S, Kamalinejad M (2000) The effects of Plantago ovata on humoral immune responses in experimental animals. J Ethnopharmacol 72: 283-286

Samiulla DS, Prashanth D, Amit A (2001) Mast-cell stabilizing activity of Bacopa monnieri. Fitoterapia 72:284-285

Saraf MN, Patwardhan BK (1988a) Pharmacological studies on Sarcostemma brevistigma. Part I Anti-allergic activity. Ind Drugs 26:49-53

Saraf MN, Patwardhan BK (1988b) Pharmacological studies on Sarcostemma brevistigma. Part II Bronchodilator activity. Ind Drugs 26:54-57

Sayah ME, Filho VC, Yunes RA, Pinheiro TR, Calixto JB (1998) Action of polygodial, a sesquiterpene isolated from Drymis winteri in the guinea-pig ileum an trachea in vitro. Eur $\mathrm{J}$ Pharmacol 344:215-221

Sen P (1993) Therapeutic potential of Tulsi (Ocimum sanctum) from experience to fact. Drug Views 1:15-18

Shin TY, Jeong HJ, Kim DK, Kim SH, Lee JK, Chae BS, Kim JH, Kang HW, Lee CM (2001a) Inhibitory action of water-soluble fraction of Terminalia chebula on systemic and local anaphylaxis. J Ethnopharmacol 74:133-140

Shin TY, Kim DK, Chae BS, Lee EJ (2001b) Antiallergic action of Magnolia officinalis on immediate hypersensitivity reaction. Arch Pharm Res 24:249-255

Shin TY, Kim SH, Lim JP, Suh ES, Jeong HJ, Kim BD, Park EJ, Hwang WJ, Rye DG, Baek SH, An NH, Kim HM (2000) Effect of Vitex rotundifolia on immediate-type allergic reaction. J Ethnopharmacol 72:443-450

Shin TY, Kim SH, Suk K, Ha JH, Kim I, Lee MG, Jun CD, Kim SY, Lim JP, Eun JS, Shin HY, Kim HM (2005) Anti-allergic effects of Lycopus lucidus on mast cellmediated allergy model. Toxicol Appl Pharmacol 209:255-262

Shin TY, Park JH, Kim HM (1999) Effect of Cryptotympana atrata extract on compound 48/80 induced anaphylactic reactions. J Ethnopharmacol 66:319-325

Shinde UA, Phadke AS, Kulkarni KR, Nair AM, Mungantiwar AA, Dikshit VJ, Saraf MN (1999) Mast cell stabilizing and lipoxygenase inhibiting activity of Cedrus deodara (Roxb.) wood oil. Ind J Exp Biol 37:258-261

Singh RK, Acharya SB, Bhattcharya SK (2000) Pharmacological activity of Elaeocarpus spharicus. Phytother Res 14:36-39

Singh S, Agrawal SS (1990) Broncho-relaxant activity of Belamcanda chinensis. Ind J Pharmacol 22:107-109

Singh S, Agrawal SS (1991) Anti asthmatic and anti-inflammatory activity of Ocimum sanctum. Int J Pharmacog 29:306-310

Smit HF, Kroes BH, van den Berg AJJ, van der Wal D, van den Worm E, Beukelman CJ, van Dijk H, Labadie RP (2000) Immunomodulatory 
and anti-inflammatory activity of Picrorrhiza scrophulariiflora. J Ethnopharmacol 73:101-109

Soares de Moura R, Costa SS, Jansen JM, Silva CA, Lopes CS, Bernardo-Filho M, Nascimento da Silva V, Criddle DN, Portela BN, Rubenich LM, Araujo RG, Carvalho LC (2002) Bronchodilator activity of Mikania glomerata Sprengel on human bronchi and guinea-pig trachea. J Pharm Pharmacol 54:249-256

Srivastava S, Gupta PP, Prasad R, Dixit KS, Palit G, Ali B, Mishra G, Saxena RC (1999) Evaluation of anti-allergic activity (Type I hypersensitivity) of Inula racemosa in rats. Ind J Physiol Pharmacol 43:235-241

Stuppner H, Dorsch W, Wagner H, Gropp M, Kepler P (1991) Antiasthmatic effects of Picorrhiza kurroa: inhibition of allergen and PAF induced bronchial obstruction in g.pigs by Androsin, Apocynine and structurally related compounds. Planta Med 57: A62

Subramoniam A, Evans DA, Valsaraj R, Rajasekharan S, Pushpangadan P (1999) Inhibition of antigen-induced degranulation of sensitized mast cells by Trichopus zeylanicus in mice and rats. $\mathrm{J}$ Ethnopharmacol 68:137-143

Suresh Kumar, R.N. Dwivedi and G. N. Chaturvedi, (1981): Scientific Appraisal of Albizzialebbeck-Benth (Shirisha), J.NIMA, XXIII, 311-316

Suzuki M, Yoshino K, Yamamoto MM, Miyase T, Sano M (2000) Inhibitory effect of Tea catechins and o-methylated derivatives of (-) - epigallocatechin -3-O-gallate on mouse type IV allergy. J Agri Food Chem 48:5649-5653

Tamaoki J, Kondo M, Sakai N (1997) Leukotriene antagonist prevents exacerbation of asthma during reduction of high dose inhaled corticosteroids. Am J Respir Crit Care Med 155:1235-1240

Thomas G, Araujo CC, Agra MF, Diniz M (1995) Preliminary studies on the hydroalcoholic extract of the root of Cissampelos sympodialis Eichl in guinea pig tracheal strips and bronchoalveolar leucocytes. Phytother Res 9:473-477

Thomas G, Araujo CC, Duarte JC, De souza DP (1997) Bronchodilator activity of an aqueous fraction of an ethanol extract of the leaves of Cissampelos sympodialis Eichl. in the guinea pig. Phytomedicine 4:233-238

Tiwari U, Rastogi B, Thakur S, Jain S, Jain NK (2003) Studies on the immunomodulatory effects of Cleome viscosa. Indian J Pharm Sci 66:171-176

Touvay C, Eienne A, Braquet P (1985) Inhibition of antigen induced lung anaphylaxis in the guinea pig by BN 52021 a new specific
PAF-acether receptor antagonist isolated from Ginkgo biloba. Agents Actions 17:371-372

Tripathi RM, Das PK (1977) Studies on anti-asthmatic and antianaphylactic activity of Albizzia lebbeck. Ind J Pharmacol 9:189194

Tripathi RM, Sen PC, Das PK (1979) Studies on the mechanism of action of Albizzia lebbeck, an Indian indigenous drug used in the treatment of atopic allergy. J Ethnopharmacol 1:385-386

Udapa AL, Udapa SL, Guruswamy MN (1991) The possible site of anti-asthmatic action of Tylophora asthmatica on pituitaryadrenal axis in albino rats. Planta Med 57:409-413

Ueda Y, Oku H, Iinuma M, Ishiguro K (2003) Effect on blood pressure decrease in response to PAF of Impatiens textori. Biol Pharm Bull 26:1505-1507

Vazquez B, Avila G, Segura D, Escalante B (1996) Anti-inflammatory activity of extracts from Aloe vera gel. J Ethnopharmacol 55:6975

Vieira-de-Abreu A, Amendoeira FC, Gomes GS, Zanon C, Chedier LM, Figueiredo MR, Kaplan MA, Frutuosa VS, Castro-FariaNeto HC, Weller PF, Bandeira-Melo C, Bozza PT (2005) Antiallergic properties of the bromeliaceae Nidularium procerum: inhibition of eosinophil activation and influx. Int Immunopharmacol 5:1966-1974

Wang N, Yao X, Ishii R, Kitanaka S (2001) Antiallergic agents from natural sources: structures and inhibitory effects on nitric oxide production and histamine release of five novel polyacetylene glucosides from Bidens parviflora Willd. Chem Pharm Bull 49:938-942

Weinberger M (1984) The pharmacology and therapeutic use of theophylline. J Allergy Clin Immunol 73:525-540

Wenzel SE, Trudeau JB, Kaminsky DA, Cohn J, Martin RJ, Westcott JY (1995) Effect of 5-lipoxygenase inhibition on bronchoconstriction and airway inflammation in nocturnal asthma. Am J Respir Crit Care Med 152:897-905

Wu JB, Chun YT, Ebizuka Y, Sankawa V (1985) Biologically active constituents of Centipeda minima: isolation of a new sesquiterpene lactones. Chem Pharm Bull 33:4091-4094

Yang T, Jia M, Meng J, Wu H, Mei Q (2006) Immunomodulatory activity of polysaccharides isolated from Angelica sinensis. Int $\mathrm{J}$ Biol Macromol 39:179-184

Yi JM, Hong SH, Kim JH, Kim HK, Song HJ, Kim HM (2002) Effect of Acanthopanax senticosus stem on mast cell-dependant anaphylaxix. J Ethnopharmacol 79:347-352 